\title{
Una propuesta para la enseñanza de las locuciones en la clase de ELE
}

\author{
ALBA GÓMEZ GONZÁLEZ \\ Hispania, escuela de español \\ Clara UREÑA TORMO \\ Universidad de Alcalá \\ clara.urena@edu.uah.es
}

\begin{abstract}
Resumen
A pesar de los grandes avances en el ámbito de la enseñanza de la fraseología, el tratamiento que las unidades fraseológicas reciben no siempre resulta totalmente satisfactorio. En este artículo se presenta una secuencia didáctica incluida en la obra Locuciones y refranes para dar y tomar (Gómez González y Ureña Tormo, 2014) y se detalla la experiencia práctica de llevarla a la clase de ELE. Esta propuesta plantea el estudio de un grupo de locuciones relacionadas con la expresión de opiniones y valoraciones, y va dirigida a estudiantes de español de nivel B2, etapa del aprendizaje en que ya se ha alcanzado un cierto dominio del idioma. Siguiendo las bases del enfoque comunicativo, la secuencia incluye una amplia tipología de actividades, distribuidas en distintas fases, con el objetivo de que los estudiantes sean capaces de identificar, comprender y memorizar las locuciones propuestas e integrarlas en su competencia comunicativa.
\end{abstract}

Palabras clave: fraseología, locuciones, valoraciones, enfoque comunicativo.

\begin{abstract}
Despite great progress in research on teaching phraseology, phraseological units are not always treated in a satisfactory manner. This paper presents a learning unit included in the book Locuciones y refranes para dar y tomar (Gómez González y Ureña Tormo, 2014) and explains the practical experience of taking it into the classroom. This proposal is devoted to learning idioms related with giving opinion and making judgements. It is aimed at B2 level students of Spanish, a stage in learning in which students have already good command of language. According to the communicative approach of language, this learning unit offers different types of activities, organised in various parts, with the purpose of teaching students to identify, understand and memorise idioms. Moreover, students learn how to integrate idioms into their language skills.
\end{abstract}

Keywords: phraseology, idioms, judgements, communicative approach. 


\section{Introducción}

Las locuciones, popularmente conocidas con términos tan variados como modismos, frases hechas o expresiones fijas, son una clase de unidades fraseológicas, como también lo son las colocaciones (ej.: cometer un error; no rotundo; vital importancia) ${ }^{1}$, las fórmulas oracionales (ej.: buenos días; madre mía) y los refranes (ej.: en abril, aguas mil; perro ladrador, poco mordedor $)^{2}$. Las locuciones se definen como unidades lingüísticas formadas por dos o más palabras que presentan una forma fija y un significado idiomático, o lo que es lo mismo, un significado que no corresponde a la suma de los significados de sus componentes (Penadés Martínez, 2012a: 23). Así, una locución tan frecuente como meter la pata presenta una serie de restricciones formales que, entre otros aspectos, le impiden cambiar su forma a plural (meter las patas), pasarla a la voz pasiva (la pata es metida) y sustituir alguno de sus elementos por uno de significado similar (colocar la pata) ${ }^{3}$. Asimismo, su significado nada tiene que ver con el hecho de introducir la extremidad de un animal en algún lugar, sino que, como es sabido, se refiere a 'hacer o decir algo inoportuno o equivocado' (Real Academia Española, 2014).

En este artículo, las unidades lingüísticas que acabamos de presentar se abordan desde una perspectiva aplicada a su enseñanza en la clase de español. Concretamente, se ofrece una propuesta didáctica para el aprendizaje de un conjunto de locuciones; una propuesta que, junto con otras, queda recogida en un material didáctico más amplio, titulado Locuciones y Refranes para dar y tomar. El libro para aprender más de 120 locuciones y refranes del español (Gómez González y Ureña Tormo, 2014). Para empezar, se presenta la motivación que justifica la elección de la enseñanza de las locuciones en ELE como tema de este artículo. Seguidamente, se detallan las características de la propuesta didáctica, con referencias explícitas a las actividades que la componen, y se ofrecen algunas consideraciones sobre su aplicación al aula de ELE. Por último, se recogen las ideas más destacadas sobre lo expuesto y se concluye con unas reflexiones finales.

\section{La enseñanza de las locuciones en ELE}

En nuestras investigaciones desarrolladas como estudiantes de posgrado (Gómez González, 2014 y Ureña Tormo, 2014), primero, y en nuestra experiencia como docentes, más tarde, hemos podido comprobar que la enseñanza de las unidades fraseológicas en el ámbito de ELE recibe un tratamiento que no siempre es el más adecuado. Especialistas en la materia, como González Rey (2012) y Penadés Martínez (2012b, 2015, 2017), se muestran conscientes de la mejora que la situación ha

\footnotetext{
${ }^{1}$ De un tiempo a esta parte, la consideración de las colocaciones como una clase de unidad fraseológica y la inclusión de su estudio en el ámbito de la fraseología es objeto de considerable debate (véase Penadés Martínez, 2017: 329).

${ }^{2}$ Esta clasificación de las unidades fraseológicas en cuatro clases ha sido tomada de Penadés Martínez (2012a: 10), una propuesta basada en la de Corpas Pastor (1996: 50-52). En la actualidad, los términos que se prefieren para designar el tipo de unidades lingüísticas objeto de estudio de la Fraseología son los de unidad fraseológica o fraseologismo (Castillo Carballo, 2006: 3 y Penadés Martínez, 2012c: 3), si bien a la hora de llevar a cabo su enseñanza en el aula de ELE, conviene utilizar otros términos más claros e intuitivos para el estudiante, como el de combinación fija de palabras (Penadés Martínez, 2017: 314).

${ }^{3}$ Para una descripción completa de las restricciones formales que pueden presentar las locuciones, véase Zuluaga (1980: 95-120).
} 
experimentado en los últimos años, pero reconocen la necesidad de seguir trabajando para lograr, sobre todo, una mayor cohesión del material didáctico disponible en el mercado (Penadés Martínez, 2017: 351-352). Es sabido que, muchas veces, las unidades fraseológicas, en concreto las locuciones, quedan al margen de las lecciones y únicamente se abordan como elementos anecdóticos. En efecto, no resulta infrecuente encontrarlas dispersas en el manual sin una organización metodológica o al final de una unidad por cuestiones de afinidad temática. También se incluyen a menudo como un dato anecdótico a propósito de un texto o una actividad, o como parte de alguna lección dedicada al español coloquial -si bien no todas las locuciones pertenecen al registro coloquial, pues las hay formales, vulgares y neutras ${ }^{-}{ }^{4}$. En cualquier caso, en ninguna de las circunstancias indicadas, la enseñanza de estas unidades parece quedar integrada en los contenidos del curso ni trabajarse según un planteamiento fundamentado.

A pesar de lo anterior, hay razones suficientes para justificar la necesidad de enseñar las unidades fraseológicas, y por lo tanto, las locuciones en ELE. Entre estas razones, destacamos algunas de las que Penadés Martínez (2012b: 97-98), Solano Rodríguez (2007: 202) y Timofeeva (2013: 323) señalan, como la inclusión de estas unidades en dos obras de referencia para la enseñanza de las lenguas, como son el Marco común europeo de referencia para las lenguas (Consejo de Europa, 2002) y, en el caso del español, el Plan Curricular del Instituto Cervantes: Niveles de referencia para el español (Instituto Cervantes, 2006); su presencia en algunas preguntas de las pruebas DELE; la viveza y expresividad que aportan al discurso; y la trasmisión de valores culturales que comporta su enseñanza.

Justificada la necesidad de incluir la enseñanza de la fraseología en los cursos de español, queda determinar qué unidades en concreto deben enseñarse según el nivel de los estudiantes y cómo debe desarrollarse este proceso (Penadés Martínez, 2012b). En cuanto a la primera cuestión, para el caso concreto de los fraseologismos que pertenecen a la clase de las locuciones, disponemos de los tres diccionarios de Penadés Martínez (2002, 2005, 2008), donde se recogen las locuciones verbales, adverbiales, nominales y adjetivas que deben enseñarse en ELE, acompañadas de una marca indicadora del nivel al que corresponde su enseñanza. En lo que concierne al modo de proceder en la didáctica de estas unidades, somos partidarias de seguir la propuesta de esta misma autora (Penadés Martínez 2015), que plantea trabajar las locuciones de manera integrada en el resto de contenidos del currículo, de modo que no queden aisladas y descontextualizadas, un planteamiento teórico que se materializa y se amplía en la propuesta didáctica que ofrecemos a continuación.

\section{La propuesta didáctica}

\subsection{Características de la propuesta}

La propuesta que aquí se presenta está dedicada a trabajar un total de 17 locuciones de nivel B2, extraídas del estudio de Penadés Martínez (2015), cuyo objetivo principal es que el discente -en este orden-identifique, comprenda, memorice y sepa utilizar el

\footnotetext{
${ }^{4}$ Algunas de estas consideraciones pueden encontrarse ampliadas en los trabajos de López Vázquez (2010), Olímpio de Oliveira Silva (2006) y Ruiz Gurillo (2000), donde se analiza el tratamiento de las unidades fraseológicas en diversos manuales de ELE.
} 
grupo de locuciones propuestas ${ }^{5}$. Algunas actividades presentan un enfoque contrastivo español-inglés, de ahí que la propuesta vaya dirigida especialmente a estudiantes anglófonos, si bien existe la posibilidad de adaptar este tipo de actividades a otras lenguas distintas al inglés ${ }^{6}$. En cuanto a la planificación didáctica, la secuencia está pensada para desarrollarse en una duración aproximada de dos horas, siguiendo una distribución del tiempo que se detalla más abajo, según las fases en las que esta se divide. En este sentido, cabe anotar que esta planificación es meramente una guía, pues puede variar en función del contexto didáctico, que será el que determine la secuenciación. En lo que concierne al material necesario, por una parte, se precisa disponer del libro donde está incluida la propuesta -o alternativamente, tenerla fotocopiada-, y por otra parte, contar con un diccionario monolingüe de la lengua española y otro bilingüe español-inglés.

El rasgo principal y más distintivo de esta secuencia, y del material donde se incluye, claro está, es su organización metodológica. Como ha quedado indicado, se trata de una organización que plantea integrar la enseñanza de las locuciones en la del resto de contenidos curriculares. De manera general, algunos manuales de español, si bien no todos, tienden a distribuir los contenidos en los siguientes tipos: funcionales, gramaticales, léxicos y culturales, una distribución que se puede aprovechar para trabajar grupos de locuciones vinculándolas con cada uno de este tipo de contenidos ${ }^{7}$. De este modo, la propuesta debe concebirse como un complemento al manual que el profesor utiliza en el aula, con el fin de enseñar las locuciones al mismo tiempo que se enseñan otros tipos de contenidos con los que estas pueden relacionarse.

Concretamente, la secuencia elegida se titula Valoramos como locos y va dirigida a trabajar un conjunto de locuciones vinculadas al contenido funcional de expresar valoraciones, una secuencia que deberá desarrollarse de manera complementaria a las explicaciones y actividades sobre la expresión de valoraciones en español. Este planteamiento permite que la enseñanza de las locuciones seleccionadas pueda llevarse a cabo de manera paralela al resto de expresiones que se emplean para valorar en el nivel B2, entre las que el Plan Curricular del Instituto Cervantes (Instituto Cervantes, 2006) ${ }^{8}$ incluye:

- Lo veo / Lo encuentro + adjetivo / SN (ej.: Todavía no quieren hacer pública la noticia, yo lo encuentro normal, ¿tú no?).

\footnotetext{
${ }^{5}$ La identificación de estos cuatro objetivos en la enseñanza-aprendizaje de las unidades fraseológicas se basa en las propuestas de Khün (citado en Ettinger 2008: 107) y Penadés Martínez (2012b: 103).

${ }^{6}$ El carácter universal de las locuciones favorece el desarrollo de prolíficas investigaciones en el ámbito de la Fraseología contrastiva, las cuales, tomadas desde una perspectiva didáctica, pueden resultar muy ventajosas de cara a adoptar el enfoque contrastivo en la enseñanza-aprendizaje de las unidades fraseológicas, tal como ha sido puesto de manifiesto por estudiosos en la materia, como Leal Riol (2011: 30) y Penadés Martínez (2012b: 102).

${ }^{7}$ Esta es la distribución que encontramos en el manual Prisma tomado como referencia, si bien es cierto que la propuesta podría desarrollarse en paralelo a otros manuales que presentan un reparto similar, como es el caso de ELE Actual, que cuenta también con una sección de fonética; de Nuevo Avance, donde se incluye un apartado de ortografía; y de otros como Sueña o Eco, que atienden a las destrezas comunicativas.

${ }^{8}$ Concretamente, las construcciones que aportamos han sido extraídas del inventario correspondiente al punto 2.4. Valorar, dentro del apartado 5. Funciones.
} 
- Es / Me parece / Veo / Encuentro / Considero / Me resulta + adjetivo / SN... o + que + subjuntivo(ej.: Me parecería muy bien que aprovecharas esa oportunidad).

Así, el propósito principal es integrar la enseñanza de las locuciones en la de estructuras como las anteriores. Para ello, consideramos que el enfoque que mejor se adapta a esta propuesta y la fundamenta es el enfoque comunicativo, pues se espera que el estudiante sea capaz de comprender y utilizar las unidades fraseológicas en sus intercambios comunicativos reales. En consistencia con este planteamiento, distinguimos las siguientes fases de trabajo: una primera fase de calentamiento y de activación de contenidos que servirá de acercamiento al tema (15 minutos); una segunda fase de presentación de los contenidos, que en el caso que nos compete se realiza a través de un soporte escrito (30 minutos); una fase de práctica controlada, mediante la cual se pretende que se identifiquen las unidades, se comprendan y se memoricen (30 minutos); $\mathrm{y}$, para terminar, una fase de práctica semilibre o libre en la que el estudiante pone en práctica lo aprendido y reflexiona sobre ello (45 minutos).

La secuencia didáctica puede llevarse a cabo una vez que el alumno, en clases anteriores o en la misma sesión, ya ha estudiado diversas estructuras valorativas. Por tanto, es en ese punto en el que el docente introduce otras expresiones que también se utilizan para valorar, como es el caso de algunas locuciones. De acuerdo con las fases establecidas, se comienza así con una actividad 0 o de calentamiento, que tiene por objetivo atraer y motivar a los estudiantes y conectar sus conocimientos previos con los nuevos, con vistas a lograr el aprendizaje significativo defendido por Ausubel (1968).

Después de esta primera fase de calentamiento, la secuencia didáctica sigue con una tarea de comprensión lectora, que conviene realizar en dos fases: una de lectura común y otra de lectura individual, para asegurar una mayor comprensión del texto. El principal objetivo de esta actividad es que el estudiante identifique las locuciones que aparecen en el texto, a partir de la formación que ha recibido sobre este tipo de unidades previamente ${ }^{9}$. Además, es recomendable aprovechar esta lectura para la explotación de otros aspectos - gramaticales, léxicos, fonéticos, culturales- de la lengua.

\footnotetext{
${ }^{9}$ Cabe tener en cuenta que esta secuencia didáctica está pensada para llevarse a cabo tras una explicación introductoria por parte del docente sobre este tipo de unidades, en la que se intente despertar la conciencia fraseológica de los estudiantes activando su conocimiento sobre la existencia de unidades fraseológicas en su lengua materna (Solano Rodríguez 2007: 204-206) y se den a conocer los principales rasgos formales, semánticos y pragmáticos de los fraseologismos.
} 
Como cada fin de semana, el pasado domingo, mi marido y yo fuimos al campo a visitar a mis padres. El viaje es largo porque viven en un lejano pueblo, pero es un lugar idilico y nos gusta ir. Alli, tienen una casa de ensueño, la llaman "Fantastic" y la verdad es que el nombre no puede ser más apropiado: es preciosa. Siempre lo pasamos de fábula, nos relajamos y nos olvidamos de todo.

Cuando llegamos, mi madre había preparado unos dulces de rechupete, de esos que tanto nos gustan. Ramón se comió prácticamente toda la bandeja, y es que mi marido tiene un estómago que es de aúpa. Así, nos recuperamos rápidamente del cansancio del viaje pero, cuando nos disponíamos a dar un paseo, sorprendentemente, se puso a llover. La verdad es que hacía mucho viento y llovía bastante. Vamos, un día de perros, ideal para quedarse en casa.

sin embargo, decidimos ir al bar del pueblo vecino. Es uno de mis rincones preferidos $y$, justamente, se estaba celebrando un concurso de baile. Allí la gente se divertía mientras se movía al ritmo de la música con una copa en la mano. Inmediatamente, nos unimos a la fiesta, que se prolongó hasta altas horas de la madrugada. Cuando salimos del bar hacia las seis de la mañana, estábamos hambrientos y decidimos ir a desayunar antes de acostarnos. Sin duda alguna, fue una noche de película.

Imagen 1. Texto para la presentación e identificación de locuciones valorativas ${ }^{10}$.

Tras el trabajo del texto, el estudiante debe reflexionar sobre si las locuciones presentan la información de manera objetiva o subjetiva, con la intención ser capaz de descubrir la función comunicativa que expresan mediante esta tarea de reflexión inductiva. Además, una vez que los estudiantes conozcan la función comunicativa de estas locuciones, un punto importante es que identifiquen con cuáles de ellas se transmite una idea positiva y con cuáles el hablante muestra una valoración negativa, actividad con la cual termina el proceso de presentación de las unidades.

El alumno ya ha asimilado el mecanismo de identificación e integración de las estructuras, de manera que está preparado para el aprendizaje de nuevas locuciones que presentan la misma función comunicativa: la valoración. Se da paso, así, a una nueva actividad para trabajar un conjunto de locuciones similares a las anteriores, entre las cuales se han de diferenciar, de nuevo, las que expresan una valoración positiva y las que transmiten una valoración negativa. Estas unidades aparecen nuevamente en contextos de habla real, pues el modelo didáctico que asienta nuestra propuesta concede un papel esencial al contexto comunicativo para determinar si un uso es o no adecuado. En este sentido, consideramos que la introducción de las locuciones, así como de cualquier otro contenido lingüístico, ha de hacerse de una manera contextualizada, ya sea mediante un texto oral o escrito.

Una vez que el estudiante ha sido expuesto a un conjunto de locuciones $\mathrm{y}$ ha reflexionado sobre su función comunicativa, la secuencia didáctica sigue con una actividad para trabajar el aspecto del significado en la que el estudiante ha de establecer relaciones de sinonimia y de significado con las unidades que acaba de estudiar. En concreto, con la información que ha obtenido hasta ahora, el aprendiente ha de identificar las locuciones que son sinónimas y, además, seleccionar su significado.

\footnotetext{
${ }^{10}$ Todas las imágenes que se incluyen en el artículo han sido extraídas de la secuencia "Valoramos como locos", del material Locuciones y Refranes para dar y tomar. El libro para aprender más de 120 locuciones y refranes del español (Gómez González y Ureña Tormo, 2014: 31-35).
} 


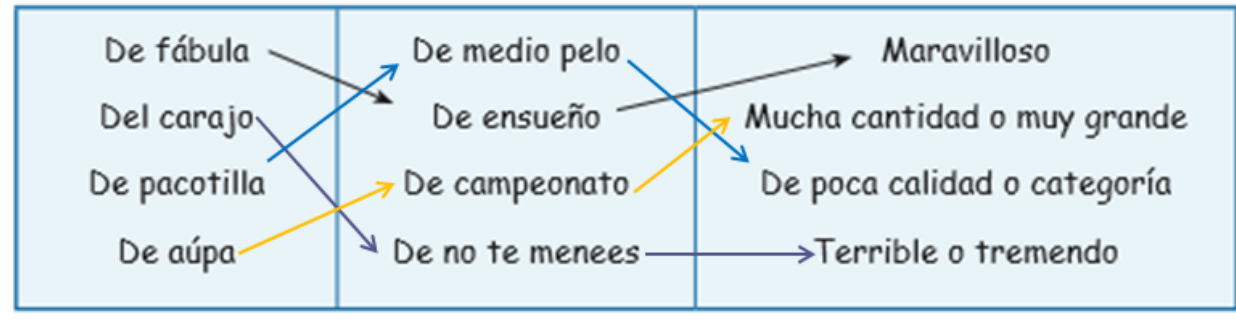

Imagen 2. Actividad para el trabajo del significado de las locuciones.

La actividad que da fin a la fase de práctica controlada es una tarea de recopilación de todas las locuciones vistas hasta el momento, con el objetivo de que los estudiantes recuerden las locuciones aprendidas y reflexionen sobre qué aspectos valora cada una de ellas. Es decir, debe indicarse si se emplean para emitir juicios valorativos acerca de personas, de lugares, de objetos, de hechos y experiencias; o bien si se usan de manera polivalente.

Llegados a este punto en que el estudiante ya conoce la forma, la función, el significado y las situaciones en que suele emplearse el conjunto de locuciones propuestas, se inicia la fase de práctica semilibre o libre con una actividad mediante la cual se prueba su capacidad de utilizarlas de manera oral a través de la interacción con un compañero. Concretamente, el alumno debe valorar distintas experiencias de su vida, como su último viaje o el último libro que ha leído, usando alguna de las locuciones aprendidas. Esta actividad, además, potencia el desarrollo de la expresión oral y, al realizarse por parejas, fomenta el establecimiento de lazos de compañerismo entre los discentes.

\begin{tabular}{|c|c|}
\hline \multicolumn{2}{|l|}{ ¿CÓMO VALORAS... } \\
\hline tu último viaje? & $\begin{array}{l}\text { El verano pasado fui a Cantabria. Hay unas playas } \\
\text { de ensueño y unos platos de rechupete, aunque } \\
\text { alli el tiempo es de perros. }\end{array}$ \\
\hline el último hotel donde te alojaste? & (1) \\
\hline el último restaurante al que fuiste? & 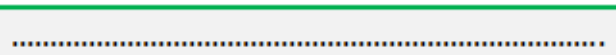 \\
\hline
\end{tabular}

Imagen 3. Actividad oral libre para la práctica de las locuciones.

Respecto al funcionamiento de esta actividad en el aula, conviene que se realice primero por parejas o en pequeños grupos $\mathrm{y}$, pasados unos minutos, que se comparta la respuesta en gran grupo. En este punto el profesor tiene un rol importante de mediador y de guía para el fomento de la participación y la conversación. Terminadas las intervenciones de los estudiantes, el profesor puede dar la retroalimentación como mejor convenga -en grupo grande, individual, etc.-, siempre que se muestre fidelidad al método que nos ocupa, de tal manera que habrá de valorarse no solo los aspectos gramaticales, sino que deberá darse prioridad a la vertiente comunicativa y funcional.

Para terminar, la secuencia finaliza con dos actividades basadas en el enfoque contrastivo, debida su reconocida y ya indicada conveniencia para el aprendizaje de los fraseologismos (ver nota 6). En la primera actividad el estudiante ha de relacionar las locuciones españolas con los equivalentes ingleses para, tras esto, reflexionar sobre la existencia de otras expresiones en su lengua que sean también equivalentes a las propuestas. Por su parte, en el segundo ejercicio los aprendices han de desarrollar una 
tarea de reflexión sobre el funcionamiento de estas y otras locuciones en cada una de las dos lenguas puestas en relación, señalando sus semejanzas y sus diferencias. Se trata de dos actividades planteadas para un trabajo individual y reflexivo del estudiante, que además pueden requerir el empleo de algunas herramientas como el diccionario, de modo que pueden resolverse fuera del aula.

\begin{tabular}{ll|}
\hline De campanillas & A hell of a \\
De butén & All fine and dandy \\
De infarto & Big name / Upper crust \\
De mil demonios & To lead a dog's life \\
De perros (llevar una vida) & Drop dead
\end{tabular}

Imagen 4. Actividad contrastiva entre las locuciones españolas y sus equivalentes en lengua inglesa.

\subsection{Puesta en práctica en el aula}

Una vez se ha descrito el desarrollo de la secuencia didáctica y se han detallado las actividades que la componen, en este apartado se señalan algunas consideraciones sobre diversas cuestiones referentes a su puesta en práctica en el aula de ELE.

En primer lugar, como se ha indicado anteriormente, esta propuesta surge con la intención de servir de complemento al manual que el profesor utiliza en sus clases, de cara a lograr una enseñanza de las locuciones que quede integrada en la del resto de contenidos que se incluyen en los manuales. En este sentido, es importante destacar que, al margen de que el manual y/o material didáctico que el docente emplee no muestre un tratamiento de las unidades fraseológicas afín al presentado en esta propuesta, o que apenas las tenga en consideración, resulta totalmente plausible adaptar el libro de texto a la situación didáctica, incluyendo y vinculando las locuciones a los contenidos que se van a tratar.

En segundo lugar, en lo que concierne a la temporalización, si bien la secuencia completa está pensada para desarrollarse en una duración aproximada de dos horas, la realidad es que el tiempo de aplicación lo marca cada contexto educativo en particular. Teniendo en cuenta los múltiples factores que pueden condicionar el ritmo de una clase, existe la posibilidad de seleccionar únicamente algunas actividades propuestas o, incluso, estas pueden ampliarse, en función de los intereses del profesor y de las necesidades de los estudiantes. Así, con un grupo de estudiantes en el que priorice el desarrollo de la expresión oral, la actividad dedicada a valorar distintas experiencias de sus vidas (ver imagen 3) puede ampliarse incluyendo nuevas vivencias, como la última película que han visto o el último concierto al que han asistido.

En tercer lugar, la puesta en práctica de esta secuencia ofrece la posibilidad al docente de promover la reflexión lingüística en los estudiantes, gracias al modelo inductivo que sigue la propuesta, y de fomentar la interacción y cooperación entre ellos, combinando distintas dinámicas de trabajo: en parejas, en pequeños grupos y en gran grupo. Además, ya no tanto desde una perspectiva pedagógica, sino afectiva y motivacional, es sabido que las unidades fraseológicas constituyen una parcela de las lenguas que suele despertar la curiosidad en los estudiantes, tanto por su aspecto idiosincrásico como por 
los valores culturales que transmiten (Leal Riol, 2011: 47 y 267). Efectivamente, según Penadés Martínez (2017: 332), el dominio de estas unidades lingüísticas genera en los estudiantes una sensación de integración en la comunidad de habla hispana, ya que acerca su discurso al de los nativos. Es este factor motivacional el que, como docentes, se recomienda aprovechar para impulsar el estudio de las unidades fraseológicas en nuestros estudiantes.

Más allá de las posibilidades que un planteamiento didáctico de estas características puede ofrecer, es necesario fijar un método de evaluación continua con el fin de que estos contenidos no caigan en el olvido de los aprendientes. De este modo, si se considera oportuno, se puede incluir alguna pregunta sobre fraseología en las pruebas o exámenes que se realizan en clase, o pueden plantearse revisiones a través de modos alternativos, de carácter lúdico, que resulten más atractivos y motivadores a los estudiantes. Por ejemplo, es posible dedicar el final de alguna sesión a realizar juegos con locuciones, como sopas de letras, el memory, el ahorcado y el dominó, los cuales nos permiten igualmente llevar un seguimiento de nivel de aprendizaje de nuestros estudiantes.

\section{Reflexiones finales}

Como ha quedado indicado al comienzo del artículo, a pesar de la evolución favorable que, con el paso de los años, ha experimentado la enseñanza de las unidades fraseológicas en ELE, todavía se encuentran ciertas deficiencias que cabría subsanar. Concretamente, en cuanto a su tratamiento en los manuales y materiales, se confirma que, pese a su longeva vida y al peso que poseen dentro de la tradición hispana, los fraseologismos, por lo general, se trabajan de una manera dispersa y aleatoria, que los desvincula del resto de contenidos académicos (López Vázquez, 2010 y Olímpio de Oliveira Silva, 2006). Esta circunstancia puede ser una de las causas por las que los propios docentes, siguiendo las programaciones establecidas, muchas veces desatendemos este campo de las lenguas que tanto interés despierta en nuestros estudiantes y cuyo aprendizaje es fundamental para el logro de su competencia comunicativa. Ante esta circunstancia, es de gran relevancia apostar por propuestas metodológicas que no solo contemplen la enseñanza de la fraseología, sino que la integren en el resto de contenidos de los programas de los cursos para evitar su aislamiento y favorecer una práctica docente que responda a unos cimientos objetivos y fundamentados.

Por todo ello, este trabajo supone, además, una llamada de atención a docentes y creadores de materiales, quienes juntos hemos de continuar esta senda. Un futuro más alentador para la enseñanza de los fraseologismos podría hacerse realidad si las planificaciones de los cursos y el diseño de material didáctico siguieran la dirección de las bases metodológicas que subyacen a la propuesta presentada. Se fomentarían, de esta manera, diversos modos de abordar la enseñanza-aprendizaje de estas unidades lingüísticas de acuerdo con unos principios rigurosos y coherentes, que velaran por la integración de este tipo de contenido en el resto de contenidos curriculares. Los beneficios de este modo de proceder podrían medirse directamente en la experiencia diaria del docente y los alumnos en el aula, así como a través de estudios empíricos que podrían constituir los cimientos de futuras investigaciones. 


\section{Bibliografía}

Ausubel, D. P. (1968). Educational Psychology: a Cognitive View. New York: Holt, Rinehart and Winston.

Borobio, V. y Palencia, R. (2012): ELE Actual B1. Curso de Español para Extranjeros. Libro del alumno. Sevilla: SM.

Castillo Carballo, Ma. A. (2006). Las colocaciones y la fraseología. Madrid: Liceus.

CONSEJO DE EuRopa (2001). Marco común europeo de referencia para las lenguas: aprendizaje, enseñanza, evaluación. Recuperado el 07/04/2017 de: http://cvc.cervantes.es/ensenanza/biblioteca_ele/marco/.

Corpas Pastor, G. (1996). Manual de fraseología española, Madrid: Gredos.

Equipo Prisma (2009). Prisma, Avanza. Nivel B2. Madrid: Edinumen.

EtTinger, S. (2008). «Alcances e límites da fraseodidáctica. Dez preguntas clave sobre o estado actual da investigación», Cadernos de Fraseoloxía Galega, 10, 95-127.

Gómez GonzÁlez, A. (2014). Más vale refrán en mano que ciento volando, Trabajo fin de máster. Alcalá de Henares: Universidad de Alcalá.

Gómez GonzÁlez, A. y Ureña Tormo, C. (2014). Locuciones y refranes para dar y tomar. El libro para aprender más de 120 locuciones y refranes del español. Niveles B2 y C1. Alcalá de Henares: Servicio de Publicaciones de la Universidad de Alcalá.

GONZÁlez REY, Ma . I. (2012). «De la didáctica de la fraseología a la fraseodidáctica», Paremia, XXI, 67-84.

Instituto Cervantes (2006). Plan curricular del Instituto Cervantes. Niveles de referencia para el español. Recuperado el 07/04/2017 de: http://cvc.cervantes.es/ensenanza/biblioteca_ele/plan_curricular/.

LEAL Riol, Ma. J. (2011). La enseñanza de la fraseología en español lengua extranjera. Estudio comparativo dirigido a estudiantes anglófonos. Valladolid: Universidad de Valladolid.

LÓPEZ VÁzQUEZ, L. (2010). «La competencia fraseológica en los textos de los manuales de ELE de nivel superior», Actas del XXI Congreso Internacional de la ASELE. Del texto a la lengua: la aplicación de los textos a la enseñanza-aprendizaje del español L2-LE, (531-542). Louvain-La-Neuve: E.M.E/ InterCommunications S.P.R.L.

Moreno, C., Moreno, V. y Zurita, P. (2010). Nuevo Avance Básico. Madrid: Sociedad General Española de Librería.

OlímPio De Oliveira Silva, Ma . E. (2006). «Fraseología y enseñanza del español como lengua extranjera», RedELE, 5. Recuperado el 20/04/2017 de: www.mec.es/redele/Biblioteca2006/OlimpiodeOliveira.shtml.

Penadés Martínez, I. (2002). Diccionario de locuciones verbales para la enseñanza del español. Madrid: Arco/Libros.

PenAdés Martínez, I. (2005). Diccionario de locuciones adverbiales para la enseñanza del español. Madrid: Arco/Libros. 
Penadés Martínez, I. (2008). Diccionario de locuciones nominales, adjetivas y pronominales para la enseñanza del español. Madrid: Arco/Libros.

Penadés Martínez, I. (2012a). Gramática y semántica de las locuciones. Alcalá de Henares: Servicio de Publicaciones de la Universidad de Alcalá.

Penadés Martínez, I. (2012b). «Didáctica de la fraseología y de la paremiología». En $\mathrm{M}^{\mathrm{a}}$. L. Ortiz Álvarez (org.), Tendências actuáis na pesquisa descritiva e aplicada em fraseologia e paremiologia. Anais. Volume I (pp. 91-117). Campinas: Pontes Editores.

Penadés Martínez, I. (2012c). «La fraseología y su objeto de estudio», Linred, X, 117. Recuperado el 09/09/2017, de: http://www.linred.es/monograficos_pdf/LR_monografico10-articulo2.pdf.

PenAdÉs MARTínez, I. (2015). «La enseñanza de la fraseología vinculada a los contenidos de los manuales de ELE». En P. Mogorrón Huerta y F. Navarro Domínguez (ed.), Fraseología, Traducción y Didáctica (pp. 241-260). Frankfurt am Main: Peter Lang.

Penadés Martínez, I. (2017). «La enseñanza de las unidades fraseológicas». En A. $\mathrm{M}^{\mathrm{a}}$. Cestero Mancera e I. Penadés Martínez (ed.), Manual del profesor de ELE (pp. 311355). Alcalá de Henares: Universidad de Alcalá.

Romero Dueñas, C. y González Hermoso, A. (2004). Eco B1. Curso modular de Español Lengua Extranjera. Libro del alumno. Madrid: Edelsa, Grupo Didascalia.

RuIz GuRILlo, L. (2000). «Un enfoque didáctico de la fraseología española para extranjeros», Espéculo. Recuperado el 20/04/2017, de: http://www.ucm.as/info/especulo/ele/fraseolo.html.

SAnz SÁnchez, B. (coord.). (2010). Sueña 3, Nueva Edición. Libro del alumno. Nivel Avanzado. Madrid: Anaya.

Solano Rodríguez, Mª A. (2007). «El papel de la conciencia fraseológica en la enseñanza y aprendizaje de una lengua extranjera». En $\mathrm{M}^{\mathrm{a}}$. I. González Rey (coord.), Les expressions figées en didactique des langues étrangères (pp. 201-221).

Ureña Tormo, C. (2014). La enseñanza de las locuciones en ELE, Trabajo fin de máster. Alcalá de Henares: Universidad de Alcalá.

Zuluaga, A. (1980). Introducción al estudio de las expresiones fijas. Frankfurt am Main: Peter Lang. 